\title{
Métodos alternativos de fungicidas para control de Botrytis Cinerea en fresa (Fragaria Vesca)
}

\section{Alternative methods of fungicides for control of Botrytis Cinerea in strawberry (Fragaria Vesca)}

Nelly del Pilar Pazmiño Miranda

Universidad Técnica de Ambato, Ecuador

Autor para correspondencia: nd.pazmino@uta.edu.ec

Fecha de recepción: 15 de diciembre 2017 - Fecha de aceptación: 26 de febrero de 2018

\section{Resumen}

El estudio evalúa los métodos alternativos para controlar Botrytis cinerea en cultivos de fresa (Fragaria vesca) con el aprovechamiento de los extractos vegetales de canela (Cinnamomum zeylanicum) y cola de caballo (Equisetum arvense L.). La investigación se realizó en un cultivo establecido de fresa de 8 meses de edad, variedad Albión, aplicando los extractos obtenidos por el método de destilación por arrastre de vapor. Las dosis evaluadas fueron $250 \mathrm{ml} / \mathrm{lt}, 500 \mathrm{ml} / \mathrm{lt}$, $750 \mathrm{ml} / \mathrm{lt}$ y $1000 \mathrm{ml} / \mathrm{lt}$ respectivamente, midiendo el efecto inhibitorio de B. cinerea en el campo a los 40 días de la primera aplicación. Las variables analizadas fueron severidad, incidencia y peso del fruto, encontrando un mejor efecto fungicida con extracto de canela con una dosis de $1000 \mathrm{ml} / \mathrm{lt}$, siendo una nueva alternativa para el control fúngico aprovechando el uso de los metabolitos secundarios de las plantas y teniendo un efecto menos nocivo en la contaminación ambiental que no afecta la salud del agricultor

Palabras claves: canela; cola de caballo; zeylanicum; equisetum; extractos vegetales

\begin{abstract}
The study evaluates alternative methods to control Botrytis cinerea in strawberry crops (Fragaria vesca) with the use of plant extracts of cinnamon (Cinnamomum zeylanicum) and horsetail (Equisetum arvense L.). The research carried out in an established strawberry culture of 8 months of age, Albion variety, applying the extracts obtained by the steam distillation method. The doses evaluated were $250 \mathrm{ml} /$ liter, $500 \mathrm{ml} /$ liter, $750 \mathrm{ml} /$ liter and $1000 \mathrm{ml} /$ liter respectively, measuring the inhibitory effect of B. cinerea in the field 40 days after the first application. The analyzed variables were severity, incidence and weight of the fruit, finding a better fungicidal effect with cinnamon extract with a dose of $1000 \mathrm{ml} /$ liter, being a new alternative for fungal control taking advantage of the use of the secondary metabolites of the plants and having a less harmful effect on environmental pollution that does not affect the health of the farmer.
\end{abstract}

Key words: cinnamon; horsetail; zeylanicum; equisetum; vegetable extracts 


\section{Introducción}

Botrytis cinerea es el agente causal de la podredumbre gris en plantas frutales como el cultivo de fresa, presentando altos porcentajes de pérdidas económicas para el agricultor (Flores, Chico, \& Cerna, 2015). Muchos agricultores ecuatorianos usan una gran cantidad de agroquímicos como carboxamidas, bencimidazoles y anilinopirimidinas (Vivienne et al., 2012) para el control de Botrytis cinerea y esto genera resistencia a las plantas (Jiménez \& Mosquera, 2014).

Siendo necesario utilizar métodos alternativos para el control de este hongo como es el aprovechamiento de los metabolitos secundarios de las plantas (Sánchez-León, Vargas-Rincón, \& Jiménez, 2015), se ha demostrado que la canela tiene un gran efecto bactericida (Narváez, 2006) y la cola de caballo contiene hasta un $4 \%$ de silicio, teniendo un efecto inhibitorio sobre los hongos que atacan a las plantas (Raya-Pérez \& Aguirre, 2012)

Investigaciones demuestran que el uso de la canela para el control de Botrytis cinerea es efectiva en plantaciones de fresa a dosis de $15 \mathrm{ml} / \mathrm{lt}$ obteniendo el extracto mediante maceración hidroetanólica llegando a controlar hasta un 24,43\% con una frecuencia de 6 días (Pazmiño, Velástegui, Curay, Yánez, \& Vásquez, 2017).

Ensayos en plantas de lechuga demuestran la eficiencia del extracto de cola de caballo en el control de Botrytis cinerea y Bremia lactucae a dosis del 500\% es decir 50g de planta entera en $10 \mathrm{ml}$ de agua destilada, utilizando el método de maceración (Tayupanta, 2012).

Por lo que la presente investigación pretende obtener métodos alternativos de fungicidas aprovechando el uso de metabolitos secundarios de las plantas de canela y cola de caballo en el control de Botrytis cinerea obteniendo nuevas alternativas agroecológicas que permitan disminuir el uso excesivo de fungicidas.

\section{Métodos}

Se investigó en una plantación de fresa variedad Albión ubicada en la Facultad de Ciencias Agropecuarias de la Universidad Técnica de Ambato, el cultivo fue establecido de 8 meses de edad, el mismo que se encontró infestado de Botrytis cinerea antes del inicio del ensayo.

Preparación de los fungicidas. Tanto para la cola de caballo como para la canela, se pesaron $15 \mathrm{lb}$ de materia seca y se colocó en un destilador industrial con 20 litros de agua, sometiendo las muestras a destilación por arrastre de vapor durante 6 horas, obteniendo $250 \mathrm{ml}$ del fungicida. La preparación de los fungicidas se usaron de acuerdo a las dosis: 250ml/lt; $500 \mathrm{ml} / \mathrm{lt} ; 750 \mathrm{ml} / \mathrm{lt}$ y $1000 \mathrm{ml} / \mathrm{lt}$

\section{Distribución de los tratamientos en el campo}

La distribución de cada tratamiento estuvo dispuesta mediante la Figura 1. Señalando que se aplicaron los fungicidas en las 16 plantas de cada tratamiento. 


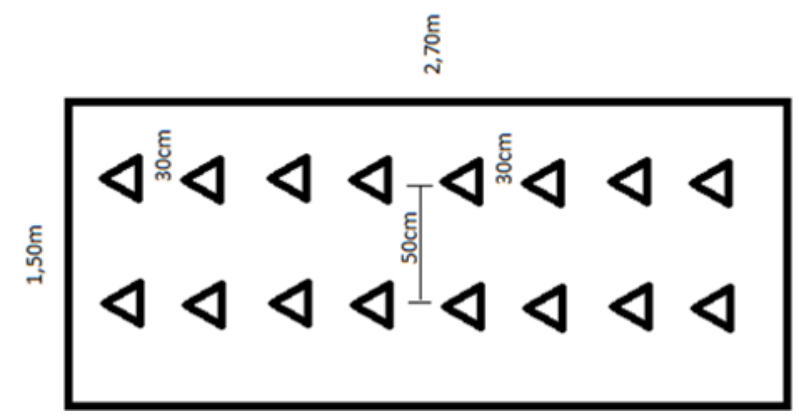

Figura 1. Esquema del ensayo de cada tratamiento

\section{Aplicación de los fungicidas}

La aspersión en las plantas se realizó cada 8 días mediante follaje, registrando datos a los 8 días, 16 días, 24 días, 32 días y 40 días.

\section{Severidad}

Se midió la severidad con la malla de puntos con una escala del 0\% a 100\%. La medición se realizó en 5 plantas seleccionadas al azar de cada tratamiento cada 8 días al momento de la cosecha y la evaluación de Botrytis cinerea fue en los frutos

\section{Incidencia}

Se evaluó en las 16 plantas de cada tratamiento mediante la ecuación1.

$$
\text { Incidencia }=\frac{\text { Número de plantas infectadas }}{\text { Número total de plantas }} * 100
$$

\section{Peso del fruto}

Se tomaron los pesos de los frutos de 5 plantas tomadas al azar en cada tratamiento, ésta determinación se realizó con la balanza analítica.

\section{Diseño experimental}

Para esta investigación se aplicó el diseño completamente al azar en arreglo factorial 2x4 + 1 (Dos extractos: canela y cola de caballo, 4 dosis: $250 \mathrm{ml} / \mathrm{lt}, 500 \mathrm{ml} / 1 \mathrm{t}, 750 \mathrm{ml} / \mathrm{lt}$ y $1000 \mathrm{ml} / \mathrm{lt}$ más un testigo al cual no se le aplicó ningún fungicida).Teniendo un total de 13 tratamientos, realizando tres repeticiones por cada tratamiento. En las variables con significación se realizó la prueba de Tukey al $95 \%$ de confianza.

\section{Análisis de los Datos}

Los datos fueron sometidos a análisis de varianza, usando el paquete estadístico Infostat 2008, en el caso de haber significación se realizaron pruebas comparativas de Tukey al 5\% 


\section{Resultados}

Se obtuvo un mejor control de Botrytis cinerea con el fungicida de canela a una dosis de $1000 \mathrm{ml} / \mathrm{lt}$ llegando a obtener una incidencia de $41,67 \%$ a los 40 días después de la primera aplicación (ver tabla 1), a diferencia del testigo cuya incidencia fue del 95,83\%, menor control B. cinerea se logra con el extracto de cola de caballo con una incidencia de 47,92\%.

Tabla 1. Porcentaje de Incidencia de Botrytis en fragaria variedad Albión

\begin{tabular}{|c|c|c|c|c|c|}
\hline \multicolumn{6}{|c|}{ Incidencia $(\%)$} \\
\hline Tratamientos & 8 Días & 16 Días & 24 Días & 32 Días & 40 Días \\
\hline Canela $1000 \mathrm{ml} / \mathrm{lt}$ & $50.00 \mathrm{a}$ & $47.92 \mathrm{a}$ & $45.83 \mathrm{a}$ & $43.75 \mathrm{a}$ & $41.67 \mathrm{a}$ \\
\hline Canela 750ml/lt & $54.17 \mathrm{a} \mathrm{b}$ & $52.08 \mathrm{a} \mathrm{b}$ & $50.00 \mathrm{a} \mathrm{b}$ & $47.92 \mathrm{a} \mathrm{b}$ & $45.83 \mathrm{a} \mathrm{b}$ \\
\hline Canela $500 \mathrm{ml} / \mathrm{lt}$ & $52.08 \mathrm{a}$ & $47.92 \mathrm{a}$ & $45.83 \mathrm{a}$ & $43.75 \mathrm{a}$ & $41.67 \mathrm{a}$ \\
\hline Canela $250 \mathrm{ml} / \mathrm{lt}$ & $56.25 \mathrm{a} \mathrm{b}$ & $54.17 \mathrm{a} \mathrm{b}$ & $52.08 \mathrm{a} \mathrm{b}$ & $50.00 \mathrm{a} \mathrm{b}$ & $47.92 \mathrm{a} \mathrm{b}$ \\
\hline Cola de Caballo $1000 \mathrm{ml} / \mathrm{lt}$ & $60.42 \mathrm{a} \mathrm{b}$ & $56.25 \mathrm{a} \mathrm{b}$ & $54.17 \mathrm{a} \mathrm{b}$ & $52.08 \mathrm{~b}$ & $47.92 \mathrm{a} \mathrm{b}$ \\
\hline Caballo 750ml/lt & $62.5 \mathrm{a} \mathrm{b}$ & $58.33 \mathrm{a} \mathrm{b}$ & $56.25 \mathrm{~b}$ & $54.17 \mathrm{~b}$ & $52.08 \mathrm{~b}$ \\
\hline Canela 500ml/lt & $62.50 \mathrm{a} \mathrm{b}$ & $60.42 b$ & $58.33 \mathrm{~b}$ & $52.08 \mathrm{~b}$ & $50.00 \mathrm{a} \mathrm{b}$ \\
\hline Caballo 250ml/lt & $66.67 \mathrm{~b}$ & $60.42 \mathrm{~b}$ & $56.25 \mathrm{~b}$ & $50.00 \mathrm{a} \mathrm{b}$ & $47.92 \mathrm{a} \mathrm{b}$ \\
\hline Testigo & $87.5 \mathrm{c}$ & $89.58 \mathrm{c}$ & $91.67 \mathrm{c}$ & $93.75 \mathrm{c}$ & $95.83 \mathrm{c}$ \\
\hline Coeficiente de Variación & 7.84 & 6.81 & 6.36 & 4.44 & 6.5 \\
\hline Error estándar & 2.78 & 2.3 & 2.08 & 1.39 & 1.96 \\
\hline p-Valor & $<0.0001$ & $<0.0001$ & $<0.0001$ & $<0.0001$ & $<0.0001$ \\
\hline
\end{tabular}

En el caso de incidencia de Botrytis cinerea sobre el cultivo de fresa, se obtiene un mejor control con el fungicida de canela a una dosis de 1000ml/lt (ver tabla2), observando que existe una relación indirecta entre el tiempo y la incidencia, pues a medida que se realizan mayores aplicaciones foliares existe una menor severidad en las plantas llegando hasta una severidad del 41,67\%, en cambio en las plantaciones testigo llega a alcanzar hasta el 95,83\% de afectación.

Tabla 2. Porcentaje de Severidad de Botrytis en fragaria variedad Albión

\begin{tabular}{llllll}
\hline \multicolumn{5}{c}{ Severidad (\%) } \\
Tratamientos & 8 Días & 16 Días & 24 Días & 32 Días & 40 Días \\
\hline Canela $1000 \mathrm{ml} / \mathrm{lt}$ & $48.6 \mathrm{a}$ & $42.11 \mathrm{a}$ & $32.85 \mathrm{a}$ & $22.08 \mathrm{a}$ & $17.74 \mathrm{a}$ \\
Canela $750 \mathrm{ml} / \mathrm{lt}$ & $50.55 \mathrm{a} \mathrm{b}$ & $42.67 \mathrm{a} \mathrm{b}$ & $35.74 \mathrm{a} \mathrm{b}$ & $26.18 \mathrm{a} \mathrm{b}$ & $21.26 \mathrm{a}$ \\
Canela $500 \mathrm{ml} / \mathrm{lt}$ & $51.83 \mathrm{~b}$ & $44.23 \mathrm{a} \mathrm{b} \mathrm{c}$ & $39.63 \mathrm{~b}$ & $32.13 \mathrm{~b} \mathrm{c}$ & $24.98 \mathrm{a} \mathrm{b}$ \\
Canela $250 \mathrm{ml} / \mathrm{lt}$ & $55.74 \mathrm{c}$ & $48.45 \mathrm{a} \mathrm{b} \mathrm{c} \mathrm{d}$ & $40.31 \mathrm{~b}$ & $32.3 \mathrm{~b} \mathrm{c}$ & $25.13 \mathrm{a} \mathrm{b}$ \\
Cola de Caballo $1000 \mathrm{ml} / \mathrm{lt}$ & $56.15 \mathrm{c}$ & $49.56 \mathrm{~b} \mathrm{c} \mathrm{d}$ & $47.59 \mathrm{c}$ & $37.31 \mathrm{c} \mathrm{d}$ & $34.3 \mathrm{~b} \mathrm{c}$ \\
Cola de Caballo $750 \mathrm{ml} / \mathrm{lt}$ & $58.6 \mathrm{~d}$ & $50.34 \mathrm{c} \mathrm{d}$ & $48.34 \mathrm{c}$ & $38.59 \mathrm{c} \mathrm{d}$ & $35.52 \mathrm{c}$ \\
Cola de Caballo 500ml/lt & $59.58 \mathrm{~d}$ & $52.57 \mathrm{~d}$ & $49.18 \mathrm{c}$ & $39.52 \mathrm{~d}$ & $36.04 \mathrm{c}$ \\
Cola de Caballo 250ml/lt & $60.85 \mathrm{~d}$ & $52.65 \mathrm{~d}$ & $50.04 \mathrm{c}$ & $41.71 \mathrm{~d}$ & $39.85 \mathrm{c}$ \\
\hline
\end{tabular}




\begin{tabular}{llllll}
\hline Testigo & $66.00 \mathrm{e}$ & $73.67 \mathrm{e}$ & $79.34 \mathrm{~d}$ & $88.34 \mathrm{e}$ & $96.67 \mathrm{~d}$ \\
Coeficiente de Variación & 1.49 & 4.81 & 5.04 & 5.97 & 9.43 \\
Error estándar & 0.49 & 1.41 & 1.37 & 1.37 & 2.01 \\
p-Valor & $<0.0001$ & $<0.0001$ & $<0.0001$ & $<0.0001$ & $<0.0001$ \\
\hline
\end{tabular}

En lo que se refiere a rendimiento los resultados (ver tabla 3) muestran que existe una mayor producción de fresas en las plantaciones que fueron aplicadas el fungicida de canela a una dosis de $100 \mathrm{ml} / \mathrm{lt}$ llegando a obtener una producción de $6664,05 \mathrm{Kg} / \mathrm{Ha}$, a diferencia de la muestra testigo en donde se obtuvieron bajos rendimientos $(1450,35 \mathrm{Kg})$.

Tabla 3. Porcentaje de Rendimiento en cultivo de fresa

\begin{tabular}{|c|c|c|c|c|c|}
\hline \multicolumn{6}{|c|}{ Rendimiento $(\mathrm{Kg} / \mathrm{Ha})$} \\
\hline Tratamientos & 8 Días & 16 Días & 24 Días & 32 Días & 40 Días \\
\hline Canela $1000 \mathrm{ml} / \mathrm{lt}$ & $3865.59 \mathrm{a}$ & $4524.52 \mathrm{a}$ & $5362.47 \mathrm{a}$ & $6336.87 \mathrm{a}$ & $6664.05 \mathrm{a}$ \\
\hline Canela 750ml/1t & $3777.99 \mathrm{a}$ & $4456.2 \mathrm{a}$ & $5045.74 \mathrm{~b}$ & $5934.51 \mathrm{a} \mathrm{b}$ & $6490.77 \mathrm{~b}$ \\
\hline Canela $500 \mathrm{ml} / \mathrm{lt}$ & $3648.4 \mathrm{~b}$ & $4384.66 \mathrm{a}$ & $4882.92 \mathrm{c}$ & $5197.69 \mathrm{c}$ & $6363.37 \mathrm{~b}$ \\
\hline Canela $250 \mathrm{ml} / \mathrm{lt}$ & $3360.98 \mathrm{c}$ & $4384.66 \mathrm{a}$ & $4372.71 \mathrm{~d}$ & $5451.97 \mathrm{~b} \mathrm{c}$ & $6202.00 \mathrm{c}$ \\
\hline Cola de Caballo $1000 \mathrm{ml} / \mathrm{lt}$ & $3089.38 \mathrm{~d}$ & $2667.84 \mathrm{~b}$ & $2947.37 \mathrm{e}$ & $3264.66 \mathrm{~d}$ & $3937.78 \mathrm{~d}$ \\
\hline Cola de Caballo 750ml/lt & $2948.4 \mathrm{e}$ & $2652.39 \mathrm{bc}$ & $2872.1 \mathrm{e}$ & $3070.76 \mathrm{~d}$ & $3752.05 \mathrm{e}$ \\
\hline Cola de Caballo 500ml/lt & $2808.82 \mathrm{f}$ & $2500.35 \mathrm{bc}$ & $2778.25 \mathrm{f}$ & $2903.83 \mathrm{~d}$ & $3390.33 \mathrm{f}$ \\
\hline Cola de Caballo 250ml/lt & $2678.11 \mathrm{~g}$ & $2141.63 \mathrm{c}$ & $2647.21 \mathrm{~g}$ & $2818.71 \mathrm{~d}$ & $3117.38 \mathrm{~g}$ \\
\hline Testigo & $2120.86 \mathrm{~h}$ & $1346.43 \mathrm{~d}$ & $1884.12 \mathrm{~h}$ & $1699.6 \mathrm{e}$ & $1450.35 \mathrm{~h}$ \\
\hline Coeficiente de Variación & 1.24 & 5.72 & 0.76 & 6.15 & 1.02 \\
\hline Error estándar & 22.55 & 106.07 & 16.08 & 144.6 & 26.97 \\
\hline p-Valor & $<0.0001$ & $<0.0001$ & $<0.0001$ & $<0.0001$ & $<0.0001$ \\
\hline
\end{tabular}

\section{Discusión}

La mejor aplicación para el control de Botrytis cinerea es con el extracto de canela a una dosis de 1000ml/lt 1 estos resultados concuerdan con (Melgarejo-Flores et al., 2013) quien establece que la canela es un potente fungicida y antioxidante natural contra Botrytis cinerea llegando a inhibir hasta el 100\% de su acción fungicida con una dosis de $5 \mathrm{~g} / \mathrm{lt}$ de aceite de canela, debiéndose su acción a la composición de aldehído cinámico el cual actúa inhibiendo las amilasas y proteasas provocando un deterioro de la pared y lisis en Botrytis cinerea (Acevedocorrea, 2017).

En lo que se refiere a incidencia de Botrytis cinerea se obtuvieron mejores resultados con el extracto de canela a una dosis de $1000 \mathrm{ml} / \mathrm{lt}$ resultados similares obtenidos con (Mohammadi, Hashemi, \& Hosseini, 2015) quien demuestra que el aceite de canela es capaz de potenciar una actividad antimicrobiana contra B. cinerea en fresa, siendo un método alternativo ecológico, y aprovechando el uso de los metabolitos secundarios de las plantas. 
Los resultados de rendimiento demuestran que existe una mayor produccion de fresas a los 40 días con $6664,05 \mathrm{~kg} / \mathrm{Ha}$, esto se debe al al contagio de las plantas por Botrytis cinerea como lo demuestran (Gonzalez, 2017) puesto que a un menor contagio existe una mayor producción y esos resultados están relacionados con la incidencia y severidad en el cultivo de fresa.

\section{Conclusión}

Se obtuvo una nueva alternativa ecológica para el control de Botrytis cinerea en plantaciones de fresa variedad Albión, usando un fungicida a base de canela con una dosis de $1000 \mathrm{ml} / \mathrm{lt}$ aprovechando así el uso de los metabolitos secundarios y dando una nueva alternativa ecológica para los agricultores, siendo necesario aprovechar el uso de los metabolitos secundarios que las diferentes plantas ofrecen para el control de plagas y enfermedades de las plantas, disminuyendo la gran cantidad de químicos usados y creando un método de control más sano que no afecte ni la salud del agricultor, ni la salud del consumidor.

\section{Agradecimientos}

Los autores agradecen a la Universidad Técnica de Ambato por el apoyo brindado durante la realización de este trabajo en el marco de ejecución del proyecto de investigación denominado "Resistencia inducida en Fragaria spp. al ataque de Tetranychus urticae Koch (Acari: Tetranychidae)"

\section{Bibliografía}

Acevedo-correa, D. (2017). Efecto antimicrobiano del clavo y la canela sobre patógenos antimicrobianos. Biotecnologia En El Sector Agropecuario Y Agroindustrial, 15(1), 5665.

Flores, W., Chico, J., \& Cerna, L. (2015). Actividad Antagónica in vitro de Clonostachys rosea sobre Fusarium oxysporum, Alternaria solani y Botrytis cinerea. Revista Rebiol, 35(1), 3442.

Gonzalez, V. (2017). Estudio y caracterización de nuevos factores de virulencia del hongo fitopatógeno Botrytis cinerea mediante técnicas moleculares. Universidad de Cádiz ( España ).

Jiménez, E. V, \& Mosquera, O. M. (2014). Actividad antifúngica In vitro de tres extractos de plantas frente a Botrytis cinerea ( Moho gris). Salud Soc Uptc. Universidad Tecnológica de Pereira, 1(2), 16-21.

Melgarejo-Flores, B. G., Ortega-Ramírez, L. A., Silva-Espinoza, B. A., González-Aguilar, G. A., Miranda, M. R. A., \& Ayala-Zavala, J. F. (2013). Antifungal protection and antioxidant enhancement of table grapes treated with emulsions, vapors, and coatings of cinnamon leaf oil. Postharvest Biology and Technology, 86, 321-328. https://doi.org/10.1016/j.postharvbio.2013.07.027 
Mohammadi, A., Hashemi, M., \& Hosseini, S. M. (2015). The control of Botrytis fruit rot in strawberry using combined treatments of Chitosan with Zataria multiflora or Cinnamomum zeylanicum essential oil. Journal of Food Science and Technology, 52(11), 7441-7448. https://doi.org/10.1007/s13197-015-1871-7

Narváez, S. (2006). Evaluación del efecto antifúngico In Vitro del aceite esencial de hoja de canela (Cinnamomum zeylanicum) puro y microencapsulado. Zamorano.

Pazmiño, P., Velástegui, G., Curay, S., Yánez, W., \& Vásquez, C. (2017). Efecto de los extractos hidro-etanólicos de canela ( Cinnamomum zeylanicum Blume ) y cola de caballo ( Equisetum arvense L .) sobre la incidencia y severidad de Botrytis cinerea en fresa Effect of hydro-ethanolic extracts of cinnamon ( Cinnamomum zeylanic. Journal of the Selva Andina Biosphere, 5(1), 29-38.

Raya-Pérez, C., \& Aguirre, C. (2012). El papel del silicio en los organismos y ecosistemas. Ciencia Y Tecnología, 22(4), 234-235. https://doi.org/10.1016/j.enfcli.2011.12.005

Sánchez-León, G., Vargas-Rincón, A., \& Jiménez, P. (2015). Evaluación de la actividad antifúngica de extractos etanólicos de dos morfotipos de Raphanus raphanistrum L. sobre tres hongos fitopatógenos. Bioagro, 27(1), 3-10.

Tayupanta, V. (2012). Control in vitro de Botrytis (Botrytis cinerea), Mildiu (Bremia lactucae) y Esclerotinia (Sclerotinia sclerotiorum) en Lechuga (Lactuca sativa), usando extractos de cola de Caballo (Equisetum arvense), Ortiga (Urtica dioica L), Ruda (Ruta graveolens) y To. Universidad Politécnica Salesiana sede Quito.

Vivienne, G., Silvana, V., Emilia, C. M., Graciela, R., Elisa, S., Pablo, G., ... Oscar, B. (2012). Resistencia a fungicidas en Botrytis cinerea en el Uruguay. Agrociencia Uruguay, 16(Mic), 97-107. 